\title{
Fermion-scalar conformal blocks
}

\author{
Luca Iliesiu, ${ }^{a}$ Filip Kos, ${ }^{b}$ David Poland, ${ }^{b, c}$ Silviu S. Pufu, ${ }^{a}$ David Simmons-Duffin ${ }^{c}$ and \\ Ran Yacoby ${ }^{a}$ \\ ${ }^{a}$ Joseph Henry Laboratories, Princeton University, \\ Washington Road, Princeton, NJ 08544, U.S.A. \\ ${ }^{b}$ Department of Physics, Yale University, \\ 217 Prospect Street, New Haven, CT 06520, U.S.A. \\ ${ }^{c}$ School of Natural Sciences, Institute for Advanced Study, \\ 1 Einstein Dr, Princeton, New Jersey 08540, U.S.A. \\ E-mail: liliesiu@princeton.edu, filip.kos@yale.edu, dpoland@gmail.com, \\ spufu@princeton.edu, davidsd@gmail.com, ranyacoby@gmail.com
}

ABSTRACT: We compute the conformal blocks associated with scalar-scalar-fermionfermion 4-point functions in 3D CFTs. Together with the known scalar conformal blocks, our result completes the task of determining the so-called 'seed blocks' in three dimensions. Conformal blocks associated with 4-point functions of operators with arbitrary spins can now be determined from these seed blocks by using known differential operators.

Keywords: Conformal and W Symmetry, Nonperturbative Effects

ARXIV EPRINT: 1511.01497 


\section{Contents}

1 Introduction 1

2 Mixed fermion-scalar conformal blocks $\quad 2$

2.1 Embedding of spinor fields 2

2.2 The three-point function 4

2.3 The four-point function 4

2.4 Recursion relations 5

2.5 Selection rules 6

3 Results $\quad 7$

3.1 Blocks in the limit $\Delta \rightarrow \infty \quad 8$

3.2 Poles and residues 8

$\begin{array}{lll}4 & \text { Summary and discussion } & 10\end{array}$

$\begin{array}{ll}\text { A The Casimir equation } & 11\end{array}$

$\begin{array}{ll}\text { B Determining OPE coefficients } & 14\end{array}$

\section{Introduction}

The conformal bootstrap [1-3] has recently resurfaced as a powerful numerical tool for constraining the algebra of local operators of conformal field theories (CFTs) in $D>2$ spacetime dimensions [4-42]. After achieving a great degree of success in constraining fourpoint functions of scalar operators in various dimensions, in [40] the numerical bootstrap was first applied to four-point functions of spin- $1 / 2$ fermions in 3D CFTs. The preliminary numerical study of [40] suggests that the fermionic bootstrap may give access to CFTs that are otherwise hard to study by analyzing only correlators of scalars, such as the Gross-Neveu-Yukawa models or the $\mathcal{N}=1$ supersymmetric Ising model.

One natural way to push this numerical program further is to study the bootstrap constraints associated with a mixed system of four-point functions of scalars and spin- $1 / 2$ fermions. Indeed, so far the numerical bootstrap has been used to place constraints on operators that appear in the operator product expansions (OPEs) of either two scalars or of two fermions. All the operators in these OPEs are bosonic. In contrast, the mixed scalarfermion bootstrap would allow us to also place constraints on the fermionic operators that appear in the OPE of a scalar with a fermion, such as the supercurrent in a supersymmetric theory. The goal of this paper is to compute the conformal blocks that are necessary in order to apply the bootstrap to such a mixed system of correlators. 
In general, the main theoretical obstruction to applying the bootstrap to four-point functions of operators with spin is that the associated conformal blocks are not always known. However, there has been some progress in our understanding of spinning conformal blocks since the original work [43-45] of Dolan and Osborn on conformal blocks of scalar four-point functions. In particular, at least in some cases it is now known that spinning conformal blocks can be obtained by acting with certain differential operators on the conformal blocks of a distinguished subset of four-point functions, which are known as "seed correlators." It is straightforward to determine these differential operators [40, 46, 47], but the seed blocks are not known in general. ${ }^{1}$

In $D \geq 4$ the full set of seed correlators is infinite, but in 3D, because of the simplicity of the Lorentz group, this set is finite. In fact, in 3D there are only two seed four-point functions: one with four scalars, and the other with two scalars and two spin- $1 / 2$ fermions. The blocks of the first seed correlator are well-known [13, 16, 27, 51], and can be used to determine the blocks corresponding to any four-point function of four bosonic or four fermionic operators using the differential operators constructed in $[40,46]$. The blocks of the second seed correlator are of two types. The first type corresponds to an exchange of a bosonic operator that appears both in the OPE of the two scalars and in the OPE of the two fermions; these blocks, too, can be obtained from the scalar conformal blocks using the differential operators in $[40,46]$. The second type of blocks corresponds to an exchange of a fermionic operator that appears in the OPE of a scalar with a fermion. These blocks are not currently known and will be determined in this paper. Using the same differential operators as in $[40,46]$, they can be used to further determine the blocks corresponding to a fermionic exchange in a four-point function of two bosonic and two fermionic operators of arbitrary spin.

As done in $[16,27]$ for scalar blocks, we will determine a recursion formula expression for our fermion-scalar blocks. We start by using the embedding space formalism, following the conventions presented in [40], to constrain the generic form of the three-point function $\left\langle\Psi\left(x_{1}\right) \Phi\left(x_{2}\right) \mathcal{O}_{\ell}(x)\right\rangle$ (section 2.2) and of the four-point function $\left\langle\Psi_{1}\left(x_{1}\right) \Phi_{2}\left(x_{2}\right) \Phi_{3}\left(x_{3}\right) \Psi_{4}\left(x_{4}\right)\right\rangle$ (section 2.3). In sections 2.4-2.5, we obtain an ansatz for a recursion formula of the conformal blocks. In section 3, we iteratively solve each order of the recursion using the Casimir equations presented in appendix A. We end with a brief discussion in section 4 that includes the decomposition of a free theory four-point function in terms of our blocks. Finally, in appendix B, we relate the coefficients that appear in the conformal block expansion to products of OPE coefficients as defined through our normalization conventions.

\section{Mixed fermion-scalar conformal blocks}

\subsection{Embedding of spinor fields}

In this section we set up our conventions for embedding 3D spinor fields into a 5D space by following [40]. We work in flat 3D spacetime with metric $\eta^{\mu \nu}=\operatorname{diag}(-1,1,1)$ and coordinates $x^{\mu}(\mu, \nu=0,1,2)$. The $3 \mathrm{D}$ conformal group $\mathrm{SO}(3,2)$ acts linearly in $5 \mathrm{D}$ embedding

\footnotetext{
${ }^{1}$ For examples of non-trivial seed blocks that are known, see [48-50].
} 
space with metric $\eta^{A B}=\eta_{A B}=\operatorname{diag}(-1,1,1,1,-1)$ and coordinates $X^{A}(A, B=0, \ldots, 4)$. We will use light-cone coordinates $X^{ \pm}=X^{4} \pm X^{3}$, and write the components of $X$ from now on as $X=\left(X^{\mu}, X^{+}, X^{-}\right)$.

The 3D spacetime is embedded in $5 \mathrm{D}$ as the projective null cone, defined to be the set of points satisfying $X \cdot X=\eta_{A B} X^{A} X^{B}=0$ and identified by $X^{A} \sim \lambda X^{A}$. The exact relation between $x^{\mu}$ and $X^{A}$ can be written as

$$
x^{\mu}=\frac{X^{\mu}}{X^{+}}, \quad X=X^{+}\left(x^{\mu}, 1, x^{2}\right),
$$

where $x^{2} \equiv \eta_{\mu \nu} x^{\mu} x^{\nu}$

Let $\mathcal{O}^{\alpha_{1} \cdots \alpha_{2 \ell}}(x)$ be a dimension $\Delta$ primary operator in the spin- $\ell$ irrep of the $\operatorname{Spin}(2,1) \simeq \operatorname{Sp}(2, \mathbf{R})$ double-cover of the $3 \mathrm{D}$ Lorentz group. ${ }^{2}$ To suppress spinor indices on operators we introduce commuting polarizations $s_{\alpha}$ and define $\mathcal{O}_{\ell}(x, s) \equiv$ $s_{\alpha_{1}} \cdots s_{\alpha_{2 \ell}} \mathcal{O}^{\alpha_{1} \cdots \alpha_{2 \ell}}(x)$. The embedding space parent of $\mathcal{O}_{\ell}(x, s)$ is given by

$$
\mathcal{O}_{\ell}(X, S) \equiv S_{I_{1}} \cdots S_{I_{2 \ell}} \mathcal{O}^{I_{1} \cdots I_{2 \ell}}(X)
$$

where $S_{I}$ are embedding space spinor polarizations transforming in the fundamental of $\operatorname{Sp}(4, \mathbf{R}) \simeq \operatorname{Spin}(3,2)$. The operator $\mathcal{O}_{\ell}(X, S)$ has the following homogeneity properties:

$$
\mathcal{O}_{\ell}(a X, b S)=a^{-(\Delta+\ell)} b^{2 \ell} \mathcal{O}_{\ell}(X, S)
$$

The projection $\mathcal{O}_{\ell}(X, S) \rightarrow \mathcal{O}_{\ell}(x, s)$ is given by the prescription

$$
\mathcal{O}_{\ell}(X, S)=\frac{1}{\left(X^{+}\right)^{\Delta}} \mathcal{O}_{\ell}(x, s)
$$

where $X$ is related to $x$ according to (2.1), and $S$ is related to $s$ through

$$
S_{I}=\sqrt{X^{+}}\left(\begin{array}{c}
s_{\alpha} \\
-x_{\gamma}^{\beta} s^{\gamma}
\end{array}\right) .
$$

Note that $S_{I}$ satisfies the transversality condition

$$
S_{I} X_{J}^{I}=0
$$

The form of correlation functions in embedding space is constrained by the $\mathrm{SO}(3,2)$ symmetry (which acts linearly on the embedding coordinates), homogeneity (2.3), and transversality (2.6).

\footnotetext{
${ }^{2} \mathrm{Sp}(2, \mathbf{R})$ indices $\alpha, \beta=1,2$ are raised and lowered with the symplectic form $\Omega_{\alpha \beta}=\Omega^{\alpha \beta}=\left(\begin{array}{cc}0 & 1 \\ -1 & 0\end{array}\right)$ and are suppressed in contractions using the convention $\psi \chi \equiv \psi_{\alpha} \chi^{\alpha}$. We turn vectors into bi-spinors using $x^{\alpha}{ }_{\beta} \equiv$ $x^{\mu}\left(\gamma_{\mu}\right)^{\alpha}{ }_{\beta}$, where $\gamma^{\mu} \equiv\left(i \sigma^{2}, \sigma^{1}, \sigma^{3}\right)$. Similarly, embedding $\operatorname{Sp}(4, \mathbf{R})$ spinor indices, $I, J=1, \ldots, 4$, are raised and lowered with $\Omega_{I J}=\Omega^{I J}=\left(\begin{array}{cc}0 & \nVdash \\ -\nVdash & 0\end{array}\right)$, and contracted as $\Psi \Lambda \equiv \Psi_{I} \Lambda^{I}$. Embedding vectors $X^{A}$ are written as embedding bi-spinors defined by $X^{I}{ }_{J} \equiv X^{A}\left(\Gamma_{A}\right)^{I}{ }_{J}$, where $\Gamma^{A} \equiv\left(\gamma^{2} \otimes \gamma^{0}, \nVdash \otimes \gamma^{1}, \nVdash \otimes \gamma^{2}, \gamma^{0} \otimes \gamma^{0}, \gamma^{1} \otimes \gamma^{0}\right)$.
} 


\subsection{The three-point function}

Consider the 3-point function of a spin- $\frac{1}{2}$ fermion primary $\Psi\left(X_{1}, S_{1}\right)$ of dimension $\Delta_{1}$, a scalar $\Phi\left(X_{2}\right)$ of dimension $\Delta_{2}$, and a third fermion $\mathcal{O}_{\ell}\left(X_{3}, S_{3}\right)$ of half-odd integer spin $\ell$ and dimension $\Delta_{3}$. Conformal invariance restricts the form of this 3-point function to be

$$
\left\langle\Psi\left(X_{1}, S_{1}\right) \Phi\left(X_{2}\right) \mathcal{O}_{\Delta_{3}, \ell}\left(X_{3}, S_{3}\right)\right\rangle=\frac{\lambda_{\Psi \Phi \mathcal{O}}^{+} r_{+}+\lambda_{\Psi \Phi \mathcal{O}}^{-} r_{-}}{X_{12}^{\frac{\Delta_{1}+\Delta_{2}-\Delta_{3}-\ell+1 / 2}{2}} X_{23}^{\frac{\Delta_{2}+\Delta_{3}-\Delta_{1}+\ell-1 / 2}{2}} X_{31}^{\frac{\Delta_{3}+\Delta_{1}-\Delta_{2}+\ell+1 / 2}{2}}},
$$

where $X_{i j} \equiv-2 X_{i} \cdot X_{j}, \lambda_{\Psi \Phi \mathcal{O}}^{ \pm}$are independent OPE coefficients, and where the structures $r_{ \pm}$can be chosen such that

$$
\begin{aligned}
& r_{+}=\frac{\left\langle S_{1} S_{3}\right\rangle\left\langle S_{3} X_{1} X_{2} S_{3}\right\rangle^{\ell-\frac{1}{2}}}{X_{12}^{\ell-\frac{1}{2}}}, \\
& r_{-}=\frac{X_{13}^{1 / 2}\left\langle S_{1} X_{2} S_{3}\right\rangle\left\langle S_{3} X_{1} X_{2} S_{3}\right\rangle^{\ell-\frac{1}{2}}}{X_{23}^{1 / 2} X_{12}^{\ell}} .
\end{aligned}
$$

As in [40], the angle-brackets are defined by

$$
\left\langle S_{1} X_{2} X_{3} \ldots S_{n}\right\rangle \equiv S_{1 I} X_{2}{ }^{I}{ }_{J} X_{3}{ }^{J}{ }_{K} \ldots \Omega^{L M} S_{n M} .
$$

The function multiplying $\lambda_{\Psi \Phi \mathcal{O}}^{+}$is even under parity, $X_{k} \rightarrow-X_{k}$, while the one multiplying $\lambda_{\Psi \Phi \mathcal{O}}^{-}$is odd. Throughout the rest of this paper, we will use \pm indices to track the parity of the 3-point structures, of the corresponding OPE coefficients, or of the conformal blocks. Furthermore, from unitarity, if the operators $\Psi\left(X_{1}, S_{1}\right)$ and $\mathcal{O}_{\Delta_{3}, \ell}\left(X_{3}, S_{3}\right)$ are real, then Fermi statistics requires that the OPE coefficients $\lambda_{\Psi \Phi \mathcal{O}}^{ \pm}$are pure imaginary.

Projecting down to $\mathbf{R}^{2,1}$ from embedding space, we can write

$$
\left\langle\Psi_{1}\left(x_{1}, s_{1}\right) \Phi_{2}\left(x_{2}\right) \mathcal{O}_{\Delta, \ell}\left(x_{3}, s_{3}\right)\right\rangle=\lambda_{\Psi \Phi \mathcal{O}}^{+} \mathcal{R}_{\Delta, \ell}^{+}+\lambda_{\Psi \Phi \mathcal{O}}^{-} \mathcal{R}_{\Delta, \ell}^{-},
$$

where the structures $\mathcal{R}_{\Delta, \ell}^{ \pm}$are obtained by applying the procedure described in section 2.1 to the structures in (2.7). We explicitly write $\mathcal{R}_{\Delta, \ell}^{ \pm}$in appendix B. The $\mathcal{R}_{\Delta, \ell}^{ \pm}$depend on all the quantities $\Delta_{1}, \Delta_{2}, \Delta, \ell$, but we have chosen to only highlight their dependence on $\Delta, \ell$ in order to avoid cluttering.

\subsection{The four-point function}

Let $\Psi_{1,4}$ be spin- $\frac{1}{2}$ fermions of dimension $\Delta_{1,4}$, and $\Phi_{2,3}$ be dimension $\Delta_{2,3}$ scalars. The 4 -point function of these operators can be written as

$$
\left\langle\Psi_{1}\left(X_{1}, S_{1}\right) \Phi_{2}\left(X_{2}\right) \Phi_{3}\left(X_{3}\right) \Psi_{4}\left(X_{4}, S_{4}\right)\right\rangle=\left(\frac{X_{24}}{X_{14}}\right)^{\frac{\Delta_{12}}{2}}\left(\frac{X_{13}}{X_{14}}\right)^{\frac{\Delta_{43}}{2}} \frac{\sum_{I} t_{I} g^{I}(u, v)}{X_{12}^{\frac{\Delta_{1}+\Delta_{2}}{2}} X_{34}^{{ }^{2}}},
$$

where $u$ and $v$ are conformally-invariant cross ratios defined as

$$
u \equiv \frac{X_{12} X_{34}}{X_{13} X_{24}}, \quad v \equiv \frac{X_{32} X_{14}}{X_{13} X_{24}}
$$


and where the $t_{I}(I=1, \ldots, 4)$ form a basis for the four independent tensor structures allowed by conformal invariance. We choose our basis to consist of the two parity even 4-point structures

$$
t_{1}=i \frac{\left\langle S_{1} S_{4}\right\rangle}{\sqrt{X_{14}}}, \quad t_{2}=-i \frac{\left\langle S_{1} X_{2} X_{3} S_{4}\right\rangle}{\sqrt{X_{12} X_{34} X_{23}}},
$$

and the two parity odd 4-point structures

$$
t_{3}=-i \frac{\left\langle S_{1} X_{2} S_{4}\right\rangle}{\sqrt{X_{12} X_{24}}}, \quad t_{4}=-i \frac{\left\langle S_{1} X_{3} S_{4}\right\rangle}{\sqrt{X_{13} X_{34}}}
$$

It will sometimes be convenient to use the coordinates $(r, \eta)$ introduced in [51] instead of $(u, v)$ in $(2.13)$. The relation between them is

$$
u=\frac{16 r^{2}}{\left(1+r^{2}+2 r \eta\right)^{2}}, \quad v=\frac{\left(1+r^{2}-2 r \eta\right)^{2}}{\left(1+r^{2}+2 r \eta\right)^{2}} .
$$

\subsection{Recursion relations}

The functions $g^{I}(u, v)$ can be expanded in conformal blocks as follows

$$
g^{I}(u, v)=\sum_{\mathcal{O}} \sum_{a, b= \pm} \lambda_{\Psi_{1} \Phi_{2} \mathcal{O}}^{a} \lambda_{\Psi_{4} \Phi_{3} \mathcal{O}}^{b} g_{\Delta, \ell}^{I, a b}(u, v)
$$

Here, $\Delta, \ell$ label the dimension and spin of the exchanged operator $\mathcal{O}$. Note that $\ell$ is a halfodd integer in the present case. The indices $a, b$ label three-point tensor structures (2.11). Furthermore, as described in appendix B, the OPE coefficients $\lambda_{\Psi_{i} \Phi_{j} \mathcal{O}}^{a}$ are the same as those appearing in the three-point function (2.7) for an appropriate normalization of the two point function of $\mathcal{O}$.

The conformal blocks are defined in terms of a sum over states in an individual conformal multiplet as

$$
\begin{aligned}
\frac{1}{X_{12}^{\frac{\Delta_{1}+\Delta_{2}}{2}} X_{34}^{\frac{\Delta_{3}+\Delta_{4}}{2}}} & \left(\frac{X_{24}}{X_{14}}\right)^{\frac{\Delta_{12}}{2}}\left(\frac{X_{13}}{X_{14}}\right)^{\frac{\Delta_{43}}{2}} \sum_{I, a, b} \lambda_{\Psi_{1} \Phi_{2} \mathcal{O}}^{a} \lambda_{\Psi_{4} \Phi_{3} \mathcal{O}}^{b} t_{I} g_{\Delta, \ell}^{I, a b}(u, v) \\
= & \sum_{\alpha=\mathcal{O}, P \mathcal{O}, \ldots} \frac{\left\langle\Psi_{1}\left(X_{1}, S_{1}\right) \Phi_{2}\left(X_{2}\right) \mid \alpha\right\rangle\left\langle\alpha \mid \Phi_{3}\left(X_{3}\right) \Psi_{4}\left(x_{4}, S_{4}\right)\right\rangle}{\langle\alpha \mid \alpha\rangle},
\end{aligned}
$$

where $\alpha$ runs over primaries $|\alpha\rangle=|\mathcal{O}\rangle$ and descendants $|\alpha\rangle=P|\mathcal{O}\rangle, \ldots$

This expression can be understood in terms of its analytic properties in $\Delta$. Here, we follow the discussion and notation of [52]. Poles in $\Delta$ occur when one of the descendant states $|\alpha\rangle$ becomes a primary. Indeed, this is only possible if $|\alpha\rangle$ is null (otherwise acting on $|\alpha\rangle$ with a special conformal generator would produce a state with nonvanishing norm), so that the corresponding denominator on the right-hand side of (2.18) vanishes. Descendants of $|\alpha\rangle$ are then also null states and each gives a contribution to the residue of the pole.

Specifically, there exist a differential operator $\mathcal{D}_{A}$ such that the descendant state

$$
\left|\mathcal{O}_{A}, s\right\rangle \equiv \mathcal{D}_{A} \mathcal{O}(x, s)|0\rangle
$$


becomes a null primary when $\Delta \rightarrow \Delta_{A}^{\star}$,

$$
\left\langle\mathcal{O}_{A}, s \mid \mathcal{O}_{A}, s^{\prime}\right\rangle=\left(\Delta-\Delta_{A}^{\star}\right) Q_{A}^{-1} \mathcal{I}_{\ell_{\mathcal{A}}}\left(s, s^{\prime}\right)+O\left(\left(\Delta-\Delta_{A}^{\star}\right)^{2}\right) .
$$

The operator $\mathcal{O}_{A}$ has spin $\ell_{A}$ and dimension $\Delta+n_{A}$, where $n_{A} \geq 1$ is the degree of $\mathcal{D}_{A}$ in $\partial_{x}$. Here, $\mathcal{I}_{\ell_{\mathcal{A}}}$ is a canonical tensor structure for a two-point function of operators with spin $\ell_{A}$. The constant $Q_{A}$ depends on the differential operator $\mathcal{D}_{A}$.

When $\Delta=\Delta_{A}^{\star}$, the operator $\mathcal{O}_{A}$ transforms like a primary with dimension $\Delta_{A} \equiv$ $\Delta_{A}^{\star}+n_{A}$ and spin $\ell_{A}$. Thus, its three-point function with $\Psi \Phi$ can be expressed in terms of our basis of three-point structures (2.11),

$$
\left.\mathcal{D}_{A} \mathcal{R}_{\Delta, \ell}^{a}\right|_{\Delta=\Delta_{A}^{\star}}=\left(M_{A}\right)_{b}^{a} \mathcal{R}_{\Delta_{A}, \ell_{A}}^{b} .
$$

where $\mathcal{R}_{\Delta_{A}, \ell_{A}}^{b}$ depends on the scaling dimensions of both the fermionic $(\Psi)$ and scalar $(\Phi)$ operators, while $M_{A}$ depends on the difference in scaling dimensions, $\Delta_{\Phi}-\Delta_{\Psi}$.

The residue of a conformal block at $\Delta=\Delta_{A}^{\star}$ comes from the contribution of $\mathcal{O}_{A}$ and all of its descendants (which also become null as $\Delta \rightarrow \Delta_{A}^{\star}$ ). Thus, it is proportional to a conformal block for $\mathcal{O}_{A}$. The constant of proportionality follows from (2.20) and (2.21),

$$
g_{\Delta, \ell}^{I, a b}=\frac{\left(M_{A}^{(L)}\right)_{c}^{a} Q_{A}\left(M_{A}^{(R)}\right)_{d}^{b}}{\Delta-\Delta_{A}^{\star}} g_{\Delta_{A}, \ell_{A}}^{I, c d}+O\left(\left(\Delta-\Delta_{A}^{\star}\right)^{0}\right),
$$

where the superscripts $L$ or $R$ indicate that we compute $M_{A}$ using the left three-point function $\left\langle\Psi_{1} \Phi_{2} \mathcal{O}\right\rangle$ or the right three-point function $\left\langle\Psi_{4} \Phi_{3} \mathcal{O}\right\rangle$. In our case, the left and right three-point functions involve the same kinds of operators, so the $M_{A}$ are simply related by $\Psi_{1} \Phi_{2} \leftrightarrow \Psi_{4} \Phi_{3}$. Since $M_{A}$ only depends on the difference between the dimension of the scalar and fermion operator, we can write

$$
M_{A}^{(L)}=M_{A}^{\Delta_{12}}, \quad M_{A}^{(R)}=M_{A}^{\Delta_{43}} .
$$

In the case studied here, the only poles in the conformal blocks are simple poles of the form (2.22). ${ }^{3}$ Furthermore, after stripping off a factor $r^{\Delta}$, the blocks have a finite limit as $\Delta \rightarrow \infty$. It follows that the blocks satisfy a recursion relation of the form

$$
\begin{aligned}
& g_{\Delta, \ell}^{I, a b}(r, \eta)=r^{\Delta} h_{\Delta, \ell}^{I, a b}(r, \eta), \\
& h_{\Delta, \ell}^{I, a b}(r, \eta)=h_{\infty, \ell}^{I, a b}(r, \eta)+\sum_{A} r^{n_{A}} \frac{\left(M_{A}^{(L)}\right)_{c}^{a} Q_{A}\left(M_{A}^{(R)}\right)_{d}^{b}}{\Delta-\Delta_{A}^{\star}} h_{\Delta_{A}, \ell_{A}}^{I, c d}(r, \eta) .
\end{aligned}
$$

(See $(2.16)$ for the relation between $(r, \eta)$ and the cross-ratios $(u, v)$ defined in (2.13).)

\subsection{Selection rules}

Because we have chosen our tensor structures to have definite parity, the indices $I, a, b$ satisfy selection rules, regardless of whether the CFT we are studying is invariant under parity. A parity even four-point structure $I_{+}=1,2$ can only arise from a combination

\footnotetext{
${ }^{3}$ See [52] for a detailed discussion of what kind of poles can occur in more general situations.
} 
of two parity even or two parity odd three-point structures $(a b=++,--)$. Similarly, a parity odd four-point structure $I_{-}=3,4$ can only arise from a combination of three-point structures of opposite parity $(a b=+-,-+)$. Thus, the only nonzero blocks are

$$
g_{\Delta, \ell}^{I_{+},++}, \quad g_{\Delta, \ell}^{I_{+},--}, \quad g_{\Delta, \ell}^{I_{-},-+}, \quad g_{\Delta, \ell}^{I_{-},+-}, \quad\left(I_{+}=1,2, I_{-}=3,4\right) .
$$

The coefficients $\left(M_{A}\right)_{b}^{a}$ obey additional selection rules depending on the parity properties of the differential operator $\mathcal{D}_{A}$ :

- If $\mathcal{D}_{A}$ preserves parity, then only $\left(M_{A}\right)_{+}^{+},\left(M_{A}\right)_{-}^{-}$are nonzero.

- If $\mathcal{D}_{A}$ changes parity, then only $\left(M_{A}\right)_{-}^{+},\left(M_{A}\right)_{+}^{-}$are nonzero.

For convenience, let us group the nonzero blocks into 2-vectors.

$$
\mathbf{g}_{\Delta, \ell}^{I_{+}} \equiv\left(\begin{array}{c}
g_{\Delta, \ell}^{I_{+},++} \\
g_{\Delta, \ell}^{I_{+},--}
\end{array}\right), \quad \mathbf{g}_{\Delta, \ell}^{I_{-}} \equiv\left(\begin{array}{c}
g_{\Delta, \ell}^{I_{-},+-} \\
g_{\Delta, \ell}^{I_{-},-+}
\end{array}\right) .
$$

Our recursion relation then has the form

$$
\mathbf{h}_{\Delta, \ell}^{I_{ \pm}}(r, \eta)=\mathbf{h}_{\infty, \ell}^{I_{ \pm}}(r, \eta)+\sum_{A} \frac{r^{n_{A}}}{\Delta-\Delta_{A}^{\star}} \mathbf{c}_{A}^{ \pm} \mathbf{h}_{\Delta_{A}, \ell_{A}}^{I_{ \pm}}(r, \eta)
$$

where $\mathbf{h}_{\Delta, \ell}^{I}$ is defined analogously to (2.26), and the $2 \times 2$ matrices $\mathbf{c}_{A}^{ \pm}$are

$$
\begin{aligned}
& \mathbf{c}_{A}^{+} \equiv Q_{A}\left(\begin{array}{l}
\left(M_{A}^{\Delta_{12}}\right)_{+}^{+}\left(M_{A}^{\Delta_{43}}\right)_{+}^{+}\left(M_{A}^{\Delta_{12}}\right)_{-}^{+}\left(M_{A}^{\Delta_{43}}\right)_{-}^{+} \\
\left(M_{A}^{\Delta_{12}}\right)_{+}^{-}\left(M_{A}^{\Delta_{43}}\right)_{+}^{-}\left(M_{A}^{\Delta_{12}}\right)_{-}^{-}\left(M_{A}^{\Delta_{43}}\right)_{-}^{-}
\end{array}\right), \\
& \mathbf{c}_{A}^{-} \equiv Q_{A}\left(\begin{array}{l}
\left(M_{A}^{\Delta_{12}}\right)_{+}^{+}\left(M_{A}^{\Delta_{43}}\right)_{-}^{-}\left(M_{A}^{\Delta_{12}}\right)_{-}^{+}\left(M_{A}^{\Delta_{43}}\right)_{+}^{-} \\
\left(M_{A}^{\Delta_{12}}\right)_{+}^{-}\left(M_{A}^{\Delta_{43}}\right)_{-}^{+}\left(M_{A}^{\Delta_{12}}\right)_{-}^{-}\left(M_{A}^{\Delta_{43}}\right)_{+}^{+}
\end{array}\right) .
\end{aligned}
$$

If $\mathcal{D}_{A}$ preserves parity then $\mathbf{c}_{A}^{ \pm}$will be diagonal, while if $\mathcal{D}_{A}$ changes parity then $\mathbf{c}_{A}^{ \pm}$will be anti-diagonal.

\section{Results}

Since each operator in the four-point function (2.17) is an eigenvector of the Casimir operator of the conformal group, the functions $g^{I}(r, \eta)$ will have to satisfy a set of Casimir equations which we derive in appendix A. Due to our choice of structures, the Casimir equations decouple into two sets of equations (A.10), one corresponding to parity even blocks and one to parity odd blocks.

Our strategy for computing the blocks $g^{I, a b}(r, \eta)$ is as follows: using the recursion ansatz obtained from the state-operator correspondence (2.27), which we plug into (A.10), we firstly solve for $\mathbf{h}_{\infty, \ell}^{I}(r, \eta)$ and then compute the residue matrices $\mathbf{c}_{A}^{ \pm}$to all orders in $r$. 


\subsection{Blocks in the limit $\Delta \rightarrow \infty$}

As explained in appendix A, the functions $\mathbf{h}_{\infty, \ell}^{I}$ can be determined by solving the Casimir equation in the limit $\Delta \rightarrow \infty$. The equation is automatically satisfied at order $\Delta^{2}$. The $r$-dependence of the solution is fixed by solving the equation at order $\Delta^{1}$. The $\eta$-dependence can be determined by examining the equation at order $\Delta^{0}$ and small $r$. This leaves two linearly independent solutions for $\mathbf{h}_{\infty, \ell}^{1,2}$ and two linearly independent solutions for $\mathbf{h}_{\infty, \ell}^{3,4}$. We fix the correct linear combinations by matching to the OPE limit $r \rightarrow 0, v \rightarrow 1$ in appendix B. The result is

$$
\begin{aligned}
& \mathbf{h}_{\infty, \ell}^{1}(r, \eta)=\frac{v^{\frac{1}{4}\left(\Delta_{12}+\Delta_{43}\right)}}{\sqrt{1-r^{2}} \sqrt{1+r^{2}+2 r \eta}}\left(\begin{array}{l}
\frac{-P_{\ell-1 / 2}^{(0,1)}(\eta)}{1-r}-\frac{P_{\ell-1 / 2}^{(1,0)}(\eta)}{1+r} \\
\frac{-P_{\ell-1 / 2}^{(0,1)}(\eta)}{1-r}+\frac{P_{\ell-1 / 2}^{(1,0)}(\eta)}{1+r}
\end{array}\right), \\
& \mathbf{h}_{\infty, \ell}^{2}(r, \eta)=\frac{v^{\frac{1}{4}\left(\Delta_{12}+\Delta_{43}\right)}}{\sqrt{1-r^{2}} \sqrt{1+r^{2}+2 r \eta}}\left(\begin{array}{l}
\frac{P_{\ell-1 / 2}^{(0,1)}(\eta)}{1-r}-\frac{P_{\ell-1 / 2}^{(1,0)}(\eta)}{1+r} \\
\frac{P_{\ell-1 / 2}^{(0,1)}(\eta)}{1-r}+\frac{P_{\ell-1 / 2}^{(1,0)}(\eta)}{1+r}
\end{array}\right) \\
& \mathbf{h}_{\infty, \ell}^{3}(r, \eta)=\frac{v^{\frac{1}{4}\left(\Delta_{12}+\Delta_{43}\right)}}{\sqrt{1-r^{2}} \sqrt{1+r^{2}-2 r \eta}}\left(\begin{array}{l}
\frac{P_{\ell-1 / 2}^{(0,1)}(\eta)}{1+r}-\frac{P_{\ell-1 / 2}^{(1,0)}(\eta)}{1-r} \\
\frac{P_{\ell-1 / 2}^{(0,1)}(\eta)}{1+r}+\frac{P_{\ell-1 / 2}^{(1,0)}(\eta)}{1-r}
\end{array}\right), \\
& \mathbf{h}_{\infty, \ell}^{4}(r, \eta)=\frac{v^{\frac{1}{4}\left(\Delta_{12}+\Delta_{43}\right)}}{\sqrt{1-r^{2}} \sqrt{1+r^{2}-2 r \eta}}\left(\begin{array}{l}
\frac{P_{\ell-1 / 2}^{(0,1)}(\eta)}{1+r}+\frac{P_{\ell-1 / 2}^{(1,0)}(\eta)}{1-r} \\
\frac{P_{\ell-1 / 2}^{(0,1)}(\eta)}{1+r}-\frac{P_{\ell-1 / 2}^{(1,0)}(\eta)}{1-r}
\end{array}\right),
\end{aligned}
$$

where $P_{n}^{(a, b)}(\eta)$ are Jacobi polynomials.

\subsection{Poles and residues}

The Casimir equations (A.10) can be solved order by order in a series expansion in $r$ and $\eta$. The full solution presented below was guessed based on the first few terms in this expansion. It was then verified that this guess solves the Casimir equations to a very high order in the expansion in $r$ and $\eta$.

In practice, the procedure for solving (A.10) in a series expansion and extracting the poles and residues appearing in (2.27) is as follows. A solution of (A.10) for the functions $\mathbf{h}_{\Delta, \ell}^{I_{ \pm}}=r^{-\Delta} \mathbf{g}_{\Delta, \ell}^{I_{ \pm}}$has an expansion

$$
\mathbf{h}_{\Delta, \ell}^{I_{ \pm}}(r, \eta)=\sum_{k=0}^{\infty} \sum_{j=0}^{k+\ell-\frac{1}{2}} \mathbf{a}_{k, j}^{I_{ \pm}}(\Delta, \ell) r^{k} \eta^{j}
$$

where the dependence on the external operator dimensions $\Delta_{12}$ and $\Delta_{34}$ is suppressed. The zeroth order coefficients $\mathbf{a}_{0,0}^{I_{ \pm}}(\Delta)$ are fixed by the explicit solution for $\mathbf{h}_{\infty, \ell}^{I_{ \pm}}(r, \eta)$ given in (3.1). For a given $\ell$ we solve for the coefficients $\mathbf{a}_{k, j}^{I_{ \pm}}$up to a finite order $k<\Lambda$, and rewrite the result in the form of the recursion formula (2.27). In particular, the locations 


\begin{tabular}{|c|c|c|c|c|l|}
\hline$A$ & $n_{A}$ & $\Delta_{A}^{\star}$ & $\ell_{A}$ & $\operatorname{parity}\left(\mathcal{D}_{A}\right)$ & values of $k$ \\
\hline$(1, k)$ & $k$ & $1-\ell-k$ & $\ell+k$ & + & $k=1,2, \ldots$, \\
$(2, k)$ & $k$ & $(3-k) / 2$ & $\ell$ & - & $k=1,3,5, \ldots$, \\
$(3, k)$ & $k$ & $2+\ell-k$ & $\ell-k$ & + & $k=1,2, \ldots, \ell-\frac{1}{2}$. \\
\hline
\end{tabular}

Table 1. Table of poles in $\Delta$ for the fermion-scalar conformal blocks. There are three series of poles, which we call $A=(1, k),(2, k),(3, k)$. The integer $k$ ranges over the values shown. $n_{A}$ is the level of the descendant corresponding to the pole, $\Delta_{A}^{\star}$ gives the dimension at which the pole appears, and $\ell_{A}$ is the spin of the descendant associated with the pole. parity $\left(\mathcal{D}_{A}\right)$ indicates whether the differential operator $\mathcal{D}_{A}$ preserves or changes parity.

of the poles $\Delta_{A}^{\star}$ are simply read off from the coefficients $\mathbf{a}_{k, j}^{I_{ \pm}}(\Delta, \ell)$, while the spins $\ell_{A}$ are inferred from the degree of the polynomials in $\eta$ that multiply each pole.

Following the above procedure, we found three series of poles (two infinite and one finite), in analogy with the case of scalar blocks $[16,27,52]$. They are listed in table 1 . We now describe the differential operators and residues associated with each pole. ${ }^{4}$

- First series of poles: the differential operators with label $(1, k)$ have the form

$$
\mathcal{D}_{1, k} \propto\left(s \partial_{x} s\right)^{k},
$$

where $s \partial_{x} s=s_{\alpha}\left(\partial_{x}\right)^{\alpha}{ }_{\beta} s^{\beta} .{ }^{5}$ By solving the Casimir equation, we find

$$
\begin{aligned}
Q_{1, k} & =-\frac{(-4)^{k} k\left(\frac{3}{2}+\ell\right)_{k}}{(k !)^{2}(1+\ell)_{k}}, \\
\left(M_{1, k}^{\Delta}\right)_{+}^{+} & =\left(\frac{-2 k-2 \Delta+1}{4}\right)_{k}, \quad\left(M_{1, k}^{\Delta}\right)_{-}^{-}=\left(\frac{-2 k-2 \Delta+3}{4}\right)_{k}, \\
\left(M_{1, k}^{\Delta}\right)_{-}^{+} & =\left(M_{1, k}^{\Delta}\right)_{+}^{-}=0,
\end{aligned}
$$

where the Pochhammer symbol is defined by $(a)_{n}=\Gamma(a+n) / \Gamma(a)$. The coefficients $\left(M_{1, k}^{\Delta}\right)_{-}^{+},\left(M_{1, k}^{\Delta}\right)_{-}^{+}$vanish because $\mathcal{D}_{1, k}$ is parity even. The residue matrices $\mathbf{c}_{1}^{ \pm}$are obtained by plugging the above into (2.28). They are diagonal.

- Second series of poles: the differential operators with label $(2, k)$ have the form

$$
\mathcal{D}_{2, k} \propto\left(s \partial_{x} \partial_{s}\right) \sum_{m} a_{m} \partial_{x}^{2 m}\left(\left(s \partial_{x} s\right)\left(\partial_{s} \partial_{x} \partial_{s}\right)\right)^{n-m}
$$

where the coefficients $a_{m}$ could be determined by demanding that $\mathcal{D}_{2, k} \mathcal{O}_{\Delta, \ell}$ is primary when $\Delta=\Delta_{2, k}^{\star}$. We find

$$
Q_{2, k}=\frac{4^{k} k \Gamma\left(\frac{k}{2}\right)^{2}}{2 \pi \Gamma\left(\frac{1+k}{2}\right)^{2}\left(\frac{1}{2}+\ell-\frac{k}{2}\right)_{k}\left(\frac{3}{2}+\ell-\frac{k}{2}\right)_{k}},
$$

\footnotetext{
${ }^{4}$ Because we obtained the residues from the Casimir equation, and not by explicitly constructing null descendants, we cannot immediately fix the normalization of the differential operators $\mathcal{D}_{A}$, and the corresponding values of $Q_{A}, M_{A}$. Different normalization conventions will rescale $Q_{A}, M_{A}$ in such a way that the residue is unaffected. Our convention is such that $Q_{A}, M_{A}$ are as simple as possible.

${ }^{5}$ The labels $(1, k),(2, k),(3, k)$ correspond to descendants of type I, III, II in [52].
} 


$$
\begin{aligned}
& \left(M_{2, k}^{\Delta}\right)_{-}^{+}=\left(\frac{-k+2 \ell-2 \Delta+2}{4}\right)_{\frac{k+1}{2}}\left(\frac{-k+2 \ell+2 \Delta+4}{4}\right)_{\frac{k-1}{2}}, \\
& \left(M_{2, k}^{\Delta}\right)_{+}^{-}=\left(\frac{-k-2 \ell-2 \Delta}{4}\right)_{\frac{k+1}{2}}\left(\frac{-k-2 \ell+2 \Delta+2}{4}\right)_{\frac{k-1}{2}}, \\
& \left(M_{2, k}^{\Delta}\right)_{+}^{+}=\left(M_{2, k}^{\Delta}\right)_{-}^{-}=0,
\end{aligned}
$$

where the matrix $\mathbf{c}_{2}^{ \pm}$is anti-diagonal for all values of $k$ and $\ell$.

- Third series of poles: the differential operators with label $(3, k)$ have the form

$$
\mathcal{D}_{3, k} \propto\left(\partial_{s} \partial_{x} \partial_{s}\right)^{k}
$$

We find

$$
\begin{aligned}
Q_{3, k} & =-\frac{(-4)^{k} k\left(\frac{1}{2}-k+\ell\right)_{k}}{(k !)^{2}(1-k+\ell)_{k}} \\
\left(M_{3, k}^{\Delta}\right)_{+}^{+} & =\left(\frac{-2 k-2 \Delta+3}{4}\right)_{k}, \quad\left(M_{3, k}^{\Delta}\right)_{-}^{-}=\left(\frac{-2 k-2 \Delta+1}{4}\right)_{k} \\
\left(M_{3, k}^{\Delta}\right)_{-}^{+} & =\left(M_{3, k}^{\Delta}\right)_{+}^{-}=0,
\end{aligned}
$$

where the matrix $\mathbf{c}_{3}^{ \pm}$is thus diagonal for all values of $k$ and $\ell$.

\section{Summary and discussion}

Our main result in this work is an explicit recursion relation for the conformal blocks corresponding to the exchange of a half-odd integer spin conformal primary that can be used to compute the blocks numerically. This recursion formula takes the form (2.27), with the various quantities appearing in this formula determined in section 3 .

As a check on our results, let us decompose a simple free theory four-point function into conformal blocks. Consider the theory of a free Majorana fermion $\psi$ and free scalar $\phi$ in three space-time dimensions. We have

$$
\left\langle\Psi\left(X_{1}, S_{1}\right) \Phi\left(X_{2}\right) \Phi\left(X_{3}\right) \Psi\left(X_{4}, S_{4}\right)\right\rangle=\frac{i\left\langle S_{1} S_{4}\right\rangle}{X_{14}^{3 / 2} X_{23}^{1 / 2}}=\frac{t_{1}}{X_{14} X_{23}^{1 / 2}},
$$

so that

$$
g_{\text {free }}^{1}(u, v)=\frac{u^{3 / 4}}{v^{1 / 2}}, \quad g_{\text {free }}^{2}(u, v)=0 .
$$

By matching several orders in an expansion in $r$, we find

$$
\begin{aligned}
g_{\text {free }}^{I_{+}}(u, v) & =\sum_{s=0}^{\infty} p_{s} g_{\frac{3}{2}+s, \frac{1}{2}+s}^{I_{+},++}(u, v), \quad\left(I_{+}=1,2\right), \\
p_{s} & =-4(s+1) .
\end{aligned}
$$

Thus, the free four-point function can be expanded in conformal blocks for higher spin currents

$$
J_{s}^{\mu_{1} \cdots \mu_{s}}=\psi \partial^{\mu_{1}} \cdots \partial^{\mu_{s}} \phi, \quad(s \geq 0) .
$$


Furthermore, only the parity even OPE coefficients $\lambda_{\Psi \Phi J_{s}}^{+}$are nonzero. Each coefficient is pure imaginary since $p_{s}=\left(\lambda_{\Psi \Phi J_{s}}^{+}\right)^{2}$ is negative, as expected in a unitary theory.

With the conformal blocks for mixed correlators between scalars and fermions in hand, one can now investigate the constraints that crossing symmetry and unitarity impose on the space of CFTs with both spin-0 and spin-1/2 conformal primary operators. We hope to report on such a study in a future publication.

\section{Acknowledgments}

We thank João Penedones, Emilio Trevisani, and Masahito Yamazaki for discussions. This work was supported by the US NSF under grant No. PHY-1418069 (LI, SSP, and RY), DOE grant number DE-SC0009988 (DSD), and NSF grant PHY-1350180 (FK and DP). DSD is supported in part by a William D. Loughlin Membership at the Institute for Advanced Study. DP is supported in part by a Martin A. and Helen Chooljian Founders' Circle Membership at the Institute for Advanced Study.

\section{A The Casimir equation}

In this appendix we derive the Casimir equations satisfied by the conformal blocks and solve them at large $\Delta$.

For a scalar field $\Phi(x)$ whose embedding space parent (defined on the 5D light cone) is $\Phi(X)=\frac{1}{\left(X^{+}\right)^{\Delta_{\Phi}}} \Phi(x)$, we can write the action of the 5D Lorentz generators as

$$
i\left[J^{A B}, \Phi(X)\right]=\left(X^{B} \frac{\partial}{\partial X_{A}}-X^{A} \frac{\partial}{\partial X_{B}}\right) \Phi(X) .
$$

Upon reduction to $3 \mathrm{D},(\mathrm{A} .1)$ reduces to the action of the conformal generators on the conformal primary field $\Phi(x)$ of dimension $\Delta_{\Phi}$.

For a 3D spinor field $\Psi^{\alpha}(x)$ whose embedding space parent is $\Psi^{I}(X)$, as defined in the main text through $S_{I} \Psi^{I}(X)=\frac{1}{\left(X^{+}\right)^{\Delta_{\Psi}}} s_{\alpha} \Psi^{\alpha}(x)$, the action of the conformal generators is more subtle. The subtlety comes from the fact that, because the $5 \mathrm{D}$ spinor polarizations $S_{I}$ are transverse, $S_{I} X^{I}{ }_{J}=0$, the parent spinor field $\Psi^{I}(X)$ is defined only modulo shifts of the form

$$
\Psi^{I}(X) \rightarrow \Psi^{I}(X)+X^{I} \Theta^{J}(X)
$$

where $\Theta^{J}(X)$ is an arbitrary spinor on the $5 \mathrm{D}$ light cone. To remove this ambiguity, one can define

$$
\tilde{\Psi}^{I}(X)=X_{J}^{I} \Psi^{I}(X) .
$$

The 5D $\tilde{\Psi}^{I}(X)$ does not suffer from the ambiguity in (A.2) because $X^{I}{ }_{J} X^{J}{ }_{K}=0$ on the 5D light cone. On $\tilde{\Psi}$, the 5D Lorentz generators have the usual action in the spinor representation

$$
i\left[J^{A B}, \tilde{\Psi}(X)\right]=\left(X^{B} \frac{\partial}{\partial X_{A}}-X^{A} \frac{\partial}{\partial X_{B}}+\frac{1}{4}\left[\Gamma^{A}, \Gamma^{B}\right]\right) \tilde{\Psi}(X),
$$


where we have suppressed all spinor indices. One can check that, once traced through all the definitions, eq. (A.4) implies that the 3D spinor field $\Psi^{\alpha}(x)$ is a conformal primary of dimension $\Delta_{\Psi}$.

Let us now analyze the conformal block decomposition of the mixed 4-point function $\left\langle\Psi_{1}\left(X_{1}\right) \Phi_{2}\left(X_{2}\right) \Phi_{3}\left(X_{3}\right) \Psi_{4}\left(X_{4}\right)\right\rangle$ in (2.12). Using (A.3) and (2.12)-(2.15), we can write

$$
\left\langle\tilde{\Psi}_{1}^{J}\left(X_{1}\right) \Phi_{2}\left(X_{2}\right) \Phi_{3}\left(X_{3}\right) \tilde{\Psi}_{4}^{K}\left(X_{4}\right)\right\rangle=\left(\frac{X_{24}}{X_{14}}\right)^{\frac{\Delta_{12}}{2}}\left(\frac{X_{13}}{X_{14}}\right)^{\frac{\Delta_{43}}{2}} \frac{\sum_{I}\left(\tilde{t}_{I}\right)^{J K} g^{I}(u, v)}{X_{12}^{\frac{\Delta_{1}+\Delta_{2}}{2}} X_{34}^{\Delta_{3}+\Delta_{4}}},
$$

where

$$
\begin{aligned}
& \left(\tilde{t}_{1}\right)^{J K}=-i \frac{\left(X_{1} X_{4}\right)^{J K}}{\sqrt{X_{14}}}, \quad\left(\tilde{t}_{2}\right)^{J K}=i \frac{\left(X_{1} X_{2} X_{3} X_{4}\right)^{J K}}{\sqrt{X_{12} X_{34} X_{23}}}, \\
& \left(\tilde{t}_{3}\right)^{J K}=i \frac{\left(X_{1} X_{2} X_{4}\right)^{J K}}{\sqrt{X_{12} X_{24}}}, \quad\left(\tilde{t}_{4}\right)^{J K}=i \frac{\left(X_{1} X_{3} X_{4}\right)^{J K}}{\sqrt{X_{13} X_{34}}} .
\end{aligned}
$$

Let $\left(J_{1}\right)^{K L}$ and $\left(J_{2}\right)^{K L}$ be the $5 \mathrm{D}$ Lorentz generators acting on $\tilde{\Psi}_{1}$ and $\Phi_{2}$ according to (A.4) and (A.1), respectively, and let us denote their sum squared as

$$
L^{2}=\frac{1}{2}\left(J_{1}+J_{2}\right)^{K L}\left(J_{1}+J_{2}\right)_{K L} .
$$

It can be shown that the contribution to (A.5) coming from the conformal multiplet of a primary $\mathcal{O}_{\Delta, \ell}$ must obey the eigenvalue equation

$$
\left(L^{2}-C_{\Delta, \ell}\right)\left(\left(\frac{X_{24}}{X_{14}}\right)^{\frac{\Delta_{12}}{2}}\left(\frac{X_{13}}{X_{14}}\right)^{\frac{\Delta_{43}}{2}} \sum_{I} \frac{\tilde{t}_{I} g_{\Delta, \ell}^{I}(u, v)}{X_{12}^{\frac{\Delta_{1}+\Delta_{2}}{2}} X_{34}^{\frac{\Delta_{3}+\Delta_{4}}{2}}}\right)=0,
$$

with eigenvalue

$$
C_{\Delta, \ell}=\Delta(\Delta-3)+\ell(\ell+1) .
$$

Using the explicit expressions in (A.4) and (A.1), one can write down the equations for $g_{\Delta, \ell}^{I}$ implied by (A.8) as

$$
\begin{gathered}
{\left[\left(\begin{array}{cc}
\mathcal{L}_{D}^{+} & \mathcal{L}_{A}^{+} \\
\mathcal{L}_{A}^{+} & \mathcal{L}_{D}^{+}
\end{array}\right)+\left(\begin{array}{cc}
0 & \frac{4 r\left(\Delta_{12}+\Delta_{43}\right)}{1+r^{2}-2 r \eta} \\
0 & \frac{4 r\left(\eta-2 r+r^{2} \eta\right)\left(\Delta_{12}+\Delta_{43}\right)}{\left(1+r^{2}-2 r \eta\right)^{2}}
\end{array}\right)\right]\left(\begin{array}{c}
g_{\Delta, \ell}^{1} \\
g_{\Delta, \ell}^{2}
\end{array}\right)=C_{\Delta, \ell}\left(\begin{array}{c}
g_{\Delta, \ell}^{1} \\
g_{\Delta, \ell}^{2}
\end{array}\right),} \\
{\left[\left(\begin{array}{cc}
\mathcal{L}_{D}^{-} & \mathcal{L}_{A}^{-} \\
\mathcal{L}_{A}^{-} & \mathcal{L}_{D}^{-}
\end{array}\right)+\left(\begin{array}{cc}
\frac{4 r\left(\eta+2 r+r^{2} \eta\right) \Delta_{43}}{\left(1+r^{2}+2 r \eta\right)^{2}} & -\frac{4 r \Delta_{12}}{1+r^{2}+2 r \eta} \\
-\frac{4 r \Delta_{43}}{1+r^{2}+2 r \eta} & \frac{4 r\left(\eta+2 r+r^{2} \eta\right) \Delta_{12}}{\left(1+r^{2}+2 r \eta\right)^{2}}
\end{array}\right)\right]\left(\begin{array}{l}
g_{\Delta, \ell}^{3} \\
g_{\Delta, \ell}^{4}
\end{array}\right)=C_{\Delta, \ell}\left(\begin{array}{l}
g_{\Delta, \ell}^{3} \\
g_{\Delta, \ell}^{4}
\end{array}\right),}
\end{gathered}
$$

where

$$
\begin{aligned}
\mathcal{L}_{D}^{ \pm}= & r^{2} \partial_{r}^{2}+\left(\eta^{2}-1\right) \partial_{\eta}^{2} \\
& +\left(\frac{4 r^{2} \eta\left(1-r^{2}\right)\left(\Delta_{12}+\Delta_{43}\right)}{\left(1+r^{2}-2 r \eta\right)\left(1+r^{2}+2 r \eta\right)}-\frac{r\left(1+3 r^{2}\right)}{1-r^{2}}-\frac{r\left(1-r^{2}\right)\left(1+r^{2} \mp 2 r \eta\right)}{\left(1+r^{2}+2 r \eta\right)\left(1+r^{2}-2 r \eta\right)}\right) \partial_{r} \\
& +\left(\frac{4\left(\eta^{2}-1\right)\left(r^{3}+r\right)\left(\Delta_{12}+\Delta_{43}\right)}{\left(1+r^{2}+2 \eta r\right)\left(1+r^{2}-2 \eta r\right)}+\frac{\left[3 \eta\left(1+r^{2}\right) \pm 2 r\left(4 \eta^{2}-1\right)\right]\left(1+r^{2} \mp 2 r \eta\right)}{\left(1+r^{2}+2 r \eta\right)\left(1+r^{2}-2 r \eta\right)}\right) \partial_{\eta}
\end{aligned}
$$




$$
\begin{aligned}
& +\left(\frac{3}{4}-\frac{4 r \Delta_{12} \Delta_{43}\left(\eta+2 r+r^{2} \eta\right)}{\left(1+r^{2}+2 r \eta\right)^{2}}\right), \\
\mathcal{L}_{A}^{ \pm}= & \frac{2 r^{2}}{1-r^{2}} \partial_{r} \pm \partial_{\eta},
\end{aligned}
$$

and the coordinates $r$ and $\eta$ are defined in (2.16). Note that the parity-even blocks, $g_{\Delta, \ell}^{1}$ and $g_{\Delta, \ell}^{2}$, decouple from the parity-odd blocks, $g_{\Delta, \ell}^{3}$ and $g_{\Delta, \ell}^{4}$.

At large $\Delta$, these equations can be solved as follows. From (2.24), we expect

$$
g_{\Delta, \ell}^{I}(r, \eta)=r^{\Delta}\left[h_{\infty, \ell}^{I}(r, \eta)+O(1 / \Delta, r)\right],
$$

for some functions $h_{\infty, \ell}^{I}$ independent of $\Delta$ that we now find. Note that the term denoted by $O(1 / \Delta, r)$ in (A.12) decays not only at large $\Delta$ but also at small $r$. (Recall that $n_{A} \geq 1$ in (2.24).) Plugging (A.12) into (A.10) and expanding up to order $\Delta^{1}$ at large $\Delta$, we find

$$
\begin{aligned}
& \left(\begin{array}{cc}
\mathcal{D}^{+} & \frac{2 r^{2}}{1-r^{2}} \\
\frac{2 r^{2}}{1-r^{2}} & \mathcal{D}^{+}
\end{array}\right)\left(\begin{array}{c}
h_{\infty, \ell}^{1} \\
h_{\infty, \ell}^{2}
\end{array}\right)=\left(\begin{array}{l}
0 \\
0
\end{array}\right), \\
& \left(\begin{array}{cc}
\mathcal{D}^{-} & \frac{2 r^{2}}{1-r^{2}} \\
\frac{2 r^{2}}{1-r^{2}} & \mathcal{D}^{-}
\end{array}\right)\left(\begin{array}{l}
h_{\infty, \ell}^{3} \\
h_{\infty, \ell}^{4}
\end{array}\right)=\left(\begin{array}{l}
0 \\
0
\end{array}\right),
\end{aligned}
$$

with

$$
\begin{aligned}
\mathcal{D}^{ \pm}= & 2 r \partial_{r}+2+\frac{4 r^{2} \eta\left(1-r^{2}\right)\left(\Delta_{12}+\Delta_{43}\right)}{\left(1+r^{2}-2 r \eta\right)\left(1+r^{2}+2 r \eta\right)}-\frac{r\left(1+3 r^{2}\right)}{1-r^{2}} \\
& -\frac{r\left(1-r^{2}\right)\left(1+r^{2} \mp 2 r \eta\right)}{\left(1+r^{2}+2 r \eta\right)\left(1+r^{2}-2 r \eta\right)} .
\end{aligned}
$$

These are first order ordinary differential equations in $r$. Their solution is

$$
\begin{aligned}
& h_{\infty, \ell}^{1}(r, \eta)=\frac{v^{\frac{1}{4}\left(\Delta_{12}+\Delta_{43}\right)}}{\sqrt{1-r^{2}} \sqrt{1+r^{2}+2 r \eta}}\left(\frac{-c_{1}(\eta)}{1-r}-\frac{c_{2}(\eta)}{1+r}\right), \\
& h_{\infty, \ell}^{2}(r, \eta)=\frac{v^{\frac{1}{4}\left(\Delta_{12}+\Delta_{43}\right)}}{\sqrt{1-r^{2}} \sqrt{1+r^{2}+2 r \eta}}\left(\frac{c_{1}(\eta)}{1-r}-\frac{c_{2}(\eta)}{1+r}\right), \\
& h_{\infty, \ell}^{3}(r, \eta)=\frac{v^{\frac{1}{4}\left(\Delta_{12}+\Delta_{43}\right)}}{\sqrt{1-r^{2}} \sqrt{1+r^{2}-2 r \eta}}\left(\frac{c_{3}(\eta)}{1+r}-\frac{c_{4}(\eta)}{1-r}\right), \\
& h_{\infty, \ell}^{4}(r, \eta)=\frac{v^{\frac{1}{4}\left(\Delta_{12}+\Delta_{43}\right)}}{\sqrt{1-r^{2}} \sqrt{1+r^{2}-2 r \eta}}\left(\frac{c_{3}(\eta)}{1+r}+\frac{c_{4}(\eta)}{1-r}\right),
\end{aligned}
$$

where $c_{i}(\eta)$ are integration constants that are arbitrary functions of $\eta$.

To determine $c_{i}(\eta)$, one has to expand the Casimir equations (A.10) to next-to-leading order in $1 / \Delta$, and the result to order $r^{0}$. Such an expansion makes sense because the terms denoted by $O\left(\Delta^{0}, r\right)$ in (A.12) do not contribute in this limit. We find that the $c_{i}$ 's must obey the second order differential equations

$$
\begin{aligned}
& {\left[\left(1-\eta^{2}\right) \partial_{\eta}^{2}+(1-3 \eta) \partial_{\eta}+\left(\ell+\frac{3}{2}\right)\left(\ell-\frac{1}{2}\right)\right]\left(\begin{array}{l}
c_{1}(\eta) \\
c_{3}(\eta)
\end{array}\right)=\left(\begin{array}{l}
0 \\
0
\end{array}\right)} \\
& {\left[\left(1-\eta^{2}\right) \partial_{\eta}^{2}+(1+3 \eta) \partial_{\eta}+\left(\ell+\frac{3}{2}\right)\left(\ell-\frac{1}{2}\right)\right]\left(\begin{array}{l}
c_{2}(\eta) \\
c_{4}(\eta)
\end{array}\right)=\left(\begin{array}{l}
0 \\
0
\end{array}\right)}
\end{aligned}
$$


Each differential equation in (A.16) has only one normalizable solution:

$$
\begin{array}{ll}
c_{1}(\eta)=C_{1} P_{\ell-1 / 2}^{(0,1)}(\eta), & c_{2}(\eta)=C_{2} P_{\ell-1 / 2}^{(1,0)}(\eta), \\
c_{3}(\eta)=C_{3} P_{\ell-1 / 2}^{(0,1)}(\eta), & c_{4}(\eta)=C_{4} P_{\ell-1 / 2}^{(1,0)}(\eta),
\end{array}
$$

with $C_{i}$ being arbitrary constants and $P_{n}^{(a, b)}(\eta)$ being Jacobi polynomials. In (3.1), we choose $C_{1}=C_{3}=1$ and $C_{2}=C_{4}=1$ (top entry of each two-component vector) or $C_{2}=C_{4}=-1$ (bottom entry of each two-component vector).

\section{B Determining OPE coefficients}

The OPE coefficients $\lambda_{\Psi \Phi \mathcal{O}}^{ \pm}$appearing in the three-point function (2.7) are the same as those appearing in the four-point function (2.17) only for a specific normalization of the operator $\mathcal{O}$. If we write the two point function of $\mathcal{O}$ as

$$
\left\langle\mathcal{O}^{\alpha_{1} \ldots \alpha_{2 l}}\left(x_{1}\right) \mathcal{O}^{\beta_{1} \ldots \beta_{2 l}}\left(x_{2}\right)\right\rangle=i^{2 \ell} c_{\mathcal{O}} \frac{\left(x_{12} i \sigma_{2}\right)^{\alpha_{1}\left(\beta_{1}\right.} \cdots\left(x_{12} i \sigma_{2}\right)^{\left.\left|\alpha_{2 \ell}\right| \beta_{2 \ell}\right)}}{\left|x_{12}\right|^{2 \Delta+2 \ell}}
$$

where $x_{i j}=x_{i}-x_{j}$ and $\alpha_{1}, \ldots, \alpha_{2 \ell}$ and $\beta_{1}, \ldots, \beta_{2 \ell}$ are spinor indices, our task is therefore to determine $c_{\mathcal{O}} \cdot{ }^{6}$

Recall that the 3-point function $\Psi_{1} \Phi_{2} \mathcal{O}$ is given in (2.11). Explicitly, we have

$$
\begin{aligned}
& \left\langle\Psi_{1}^{\beta}\left(x_{1}\right) \Phi_{2}\left(x_{2}\right) \mathcal{O}^{\alpha_{1} \alpha_{2} \ldots \alpha_{2 \ell}}\left(x_{3}\right)\right\rangle \\
& =\lambda_{\Psi_{1} \Phi_{2} \mathcal{O}}^{+} \frac{\left(x_{13} i \sigma_{2}\right)^{\beta\left(\alpha_{1}\right.}\left(x_{31} x_{12} x_{23} i \sigma_{2}\right)^{\alpha_{2} \alpha_{3}} \cdots\left(x_{31} x_{12} x_{23} i \sigma_{2}\right)^{\left.\alpha_{2 \ell-1} \alpha_{2 \ell}\right)}}{\left|x_{12}\right|^{\Delta_{1}+\Delta_{2}-\Delta+\ell-\frac{1}{2}}\left|x_{23}\right|^{\Delta_{2}+\Delta-\Delta_{1}+\ell-\frac{1}{2}}\left|x_{31}\right|^{\Delta+\Delta_{1}-\Delta_{2}+\ell+\frac{1}{2}}} \\
& \quad-\lambda_{\Psi_{1} \Phi_{2} \mathcal{O}}^{-} \frac{\left(x_{12} x_{23} i \sigma_{2}\right)^{\beta\left(\alpha_{1}\right.}\left(x_{31} x_{12} x_{23} i \sigma_{2}\right)^{\alpha_{2} \alpha_{3}} \cdots\left(x_{31} x_{12} x_{23} i \sigma_{2}\right)^{\left.\alpha_{2 l-1} \alpha_{2 l}\right)}}{\left|x_{12}\right|^{\Delta_{1}+\Delta_{2}-\Delta+\ell+\frac{1}{2}}\left|x_{23}\right|^{\Delta_{2}+\Delta-\Delta_{1}+\ell+\frac{1}{2}}\left|x_{31}\right|^{\Delta+\Delta_{1}-\Delta_{2}+\ell-\frac{1}{2}}} .
\end{aligned}
$$

In the OPE limit $x_{1} \rightarrow x_{2}$, the three-point function (B.2) becomes

$$
\begin{aligned}
& \left\langle\Psi_{1}^{\beta}\left(x_{1}\right) \Phi_{2}\left(x_{2}\right) \mathcal{O}^{\alpha_{1} \alpha_{2} \ldots \alpha_{2 \ell}}\left(x_{3}\right)\right\rangle \\
& \approx \lambda_{\Psi_{1} \Phi_{2} \mathcal{O}}^{+}\left(i \sigma_{2} x_{12}\right)_{\beta_{1} \beta_{2}} \cdots\left(i \sigma_{2} x_{12}\right)_{\beta_{2 \ell-2} \beta_{2 \ell-1}} \frac{\left(x_{23} i \sigma_{2}\right)^{\beta\left(\alpha_{1}\right.}\left(x_{23} i \sigma_{2}\right)^{\left|\beta_{1}\right| \alpha_{2}} \cdots\left(x_{23} i \sigma_{2}\right)^{\left.\left|\beta_{2 \ell-1}\right| \alpha_{2 \ell}\right)}}{\left|x_{12}\right|^{\Delta_{1}+\Delta_{2}-\Delta+\ell-\frac{1}{2}}\left|x_{23}\right|^{2 \Delta+2 \ell}} \\
& -\lambda_{\Psi_{1} \Phi_{2} \mathcal{O}}^{-}\left(i \sigma_{2} x_{12}\right)_{\beta_{1} \beta_{2}} \cdots\left(i \sigma_{2} x_{12}\right)_{\beta_{2 \ell-2} \beta_{2 \ell-1}} \frac{\left(x_{12} x_{23} i \sigma_{2}\right)^{\beta\left(\alpha_{1}\right.}\left(x_{23} i \sigma_{2}\right)^{\left|\beta_{1}\right| \alpha_{2}} \cdots\left(x_{23} i \sigma_{2}\right)^{\left.\left|\beta_{2 \ell-1}\right| \alpha_{2 \ell}\right)}}{\left|x_{12}\right|^{\Delta_{1}+\Delta_{2}-\Delta+\ell+\frac{1}{2}}\left|x_{23}\right|^{2 \Delta+2 \ell}} .
\end{aligned}
$$

From (B.3), we can extract the contribution of $\mathcal{O}$ to the $\Psi_{1} \times \Phi_{2}$ OPE:

$$
\begin{aligned}
& \Psi_{1}^{\beta}\left(x_{1}\right) \Phi_{2}\left(x_{2}\right) \\
& =\cdots+\left(-\frac{i^{2 \ell} \lambda_{\Psi_{1} \Phi_{2} \mathcal{O}}^{+}}{c_{\mathcal{O}}}\left(i \sigma_{2} x_{12}\right)_{\alpha_{1} \beta_{1}} \cdots\left(i \sigma_{2} x_{12}\right)_{\alpha_{\ell-\frac{1}{2}} \beta_{\ell-\frac{1}{2}}} \frac{\mathcal{O}^{\beta \alpha_{1} \beta_{1} \ldots \alpha_{\ell-\frac{1}{2}} \beta_{\ell-\frac{1}{2}}}\left(x_{2}\right)}{\left|x_{12}\right|^{\Delta_{1}+\Delta_{2}-\Delta+\ell-\frac{1}{2}}}\right. \\
& \left.\quad+\frac{i^{2 \ell} \lambda_{\Psi_{1} \Phi_{2} \mathcal{O}}^{-}}{c_{\mathcal{O}}}\left(i \sigma_{2} x_{12}\right)_{\alpha_{1} \beta_{1}} \cdots\left(i \sigma_{2} x_{12}\right)_{\alpha_{\ell-\frac{1}{2}} \beta_{\ell-\frac{1}{2}}}\left(x_{12}\right)_{\gamma}^{\beta} \frac{\mathcal{O}^{\gamma \alpha_{1} \beta_{1} \ldots \alpha_{\ell-\frac{1}{2}} \beta_{\ell-\frac{1}{2}}}\left(x_{2}\right)}{\left|x_{12}\right|^{\Delta_{1}+\Delta_{2}-\Delta+\ell+\frac{1}{2}}}\right)+\cdots
\end{aligned}
$$

\footnotetext{
${ }^{6}$ In a reflection positive theory, the OPE coefficients $\lambda_{\Psi_{i} \Phi_{j} \mathcal{O}}$ will be pure imaginary and $c_{\mathcal{O}}$ will be positive. In a theory that violates reflection positivity, our conventions are such that $c_{\mathcal{O}}$ should still be fixed to the positive value determined in this appendix, after which the coefficients $\lambda_{\Psi_{i} \Phi_{j} \mathcal{O}}$ may no longer be pure imaginary.
} 
Now we can examine the mixed 4-point function in the OPE limit. As we take $x_{3} \rightarrow x_{4}$, using (B.4) and (B.1) and the fact that eq. (2.7) implies $\lambda_{\Psi_{4} \Phi_{3} \mathcal{O}}^{b}=(-1)^{\ell-\frac{1}{2}} \lambda_{\Phi_{3} \Psi_{4} \mathcal{O}}^{b}$, one can write the four-point function as

$$
\left\langle\Psi_{1}^{\alpha}\left(x_{1}\right) \Phi_{2}\left(x_{2}\right) \Phi_{3}\left(x_{3}\right) \Psi_{4}^{\beta}\left(x_{4}\right)\right\rangle=\left(\frac{\left|x_{24}\right|}{\left|x_{14}\right|}\right)^{\Delta_{12}}\left(\frac{\left|x_{13}\right|}{\left|x_{14}\right|}\right)^{\Delta_{43}} \sum_{\mathcal{O}} \sum_{a, b= \pm} \frac{\lambda_{\Psi_{1} \Phi_{2} \mathcal{O}}^{a} \lambda_{\Psi_{4} \Phi_{3} \mathcal{O}}^{b}\left(g_{\mathcal{O}}^{a b}\left(x_{i}\right)\right)^{\alpha \beta}}{\left|x_{12}\right|^{\Delta_{1}+\Delta_{2}}\left|x_{34}\right|^{\Delta_{3}+\Delta_{4}}},
$$

with

$$
\begin{aligned}
\left(g_{\mathcal{O}}^{++}\right)^{\alpha \beta}\left(x_{i}\right)= & \left(\frac{\left|x_{14}\right|}{\left|x_{24}\right|}\right)^{\Delta_{12}}\left(\frac{\left|x_{14}\right|}{\left|x_{13}\right|}\right)^{\Delta_{43}} \frac{(-1)^{\ell+1}}{c_{\mathcal{O}}}\left(i \sigma_{2} x_{34}\right)_{\alpha_{1} \alpha_{2}} \cdots\left(i \sigma_{2} x_{34}\right)_{\alpha_{2 \ell-2} \alpha_{2 \ell-1}} \\
& \times \frac{\left(x_{23} i \sigma_{2}\right)^{\alpha(\beta}\left(x_{31} x_{12} x_{23} i \sigma_{2}\right)^{\alpha_{1} \alpha_{2}} \cdots\left(x_{31} x_{12} x_{23} i \sigma_{2}\right)^{\left.\alpha_{2 \ell-2} \alpha_{2 \ell-1}\right)}}{\left|x_{12}\right|^{-\Delta+\ell-\frac{1}{2}}\left|x_{23}\right|^{\Delta-\Delta_{12}+\ell-\frac{1}{2}}\left|x_{31}\right|^{\Delta+\Delta_{12}+\ell+\frac{1}{2}}\left|x_{34}\right|^{-\Delta+\ell-\frac{1}{2}}} \\
\left(g_{\mathcal{O}}^{--}\right)^{\alpha \beta}\left(x_{i}\right)= & \left(\frac{\left|x_{14}\right|}{\left|x_{24}\right|}\right)^{\Delta_{12}}\left(\frac{\left|x_{14}\right|}{\left|x_{13}\right|}\right)^{\Delta_{43}} \frac{(-1)^{\ell}}{c_{\mathcal{O}}}\left(i \sigma_{2} x_{34}\right)_{\alpha_{1} \alpha_{2}} \cdots\left(i \sigma_{2} x_{34}\right)_{\alpha_{2 \ell-2} \alpha_{2 \ell-1}} \\
& \times\left(x_{34}\right)_{\gamma}^{\beta} \frac{\left(x_{12} x_{23} i \sigma_{2}\right)^{\alpha(\gamma}\left(x_{31} x_{12} x_{23} i \sigma_{2}\right)^{\alpha_{1} \alpha_{2}} \cdots\left(x_{31} x_{12} x_{23} i \sigma_{2}\right)^{\left.\alpha_{2 \ell-2} \alpha_{2 \ell-1}\right)}}{\left|x_{12}\right|^{-\Delta+\ell+\frac{1}{2}}\left|x_{23}\right|^{\Delta-\Delta_{12}+\ell+\frac{1}{2}}\left|x_{31}\right|^{\Delta+\Delta_{12}+\ell-\frac{1}{2}}\left|x_{34}\right|^{-\Delta+\ell+\frac{1}{2}}} \\
\left(g_{\mathcal{O}}^{+-}\right)^{\alpha \beta}\left(x_{i}\right)= & \left(\frac{\left|x_{14}\right|}{\left|x_{24}\right|}\right)^{\Delta_{12}}\left(\frac{\left|x_{14}\right|}{\left|x_{13}\right|}\right)^{\Delta_{43}} \frac{(-1)^{\ell+1}}{c_{\mathcal{O}}}\left(i \sigma_{2} x_{34}\right)_{\alpha_{1} \alpha_{2}} \cdots\left(i \sigma_{2} x_{34}\right)_{\alpha_{2 \ell-2} \alpha_{2 \ell-1}} \\
& \times\left(x_{34}\right)_{\gamma}^{\beta} \frac{\left(x_{23} i \sigma_{2}\right)^{\alpha(\gamma}\left(x_{31} x_{12} x_{23} i \sigma_{2}\right)^{\alpha_{1} \alpha_{2}} \cdots\left(x_{31} x_{12} x_{23} i \sigma_{2}\right)^{\left.\alpha_{2 \ell-2} \alpha_{2 \ell-1}\right)}}{\left|x_{12}\right|^{-\Delta+\ell-\frac{1}{2}}\left|x_{23}\right|^{\Delta-\Delta_{12}+\ell-\frac{1}{2}}\left|x_{31}\right|^{\Delta+\Delta_{12}+\ell+\frac{1}{2}}\left|x_{34}\right|^{-\Delta+\ell+\frac{1}{2}}} \\
\left(g_{\mathcal{O}}^{-+}\right)^{\alpha \beta}\left(x_{i}\right)= & \left(\frac{\left|x_{14}\right|}{\left|x_{24}\right|}\right)^{\Delta_{12}}\left(\frac{\left|x_{14}\right|}{\left|x_{13}\right|}\right)^{\Delta_{43}} \frac{(-1)^{\ell}}{c_{\mathcal{O}}}\left(i \sigma_{2} x_{34}\right)_{\alpha_{1} \alpha_{2}} \cdots\left(i \sigma_{2} x_{34}\right)_{\alpha_{2 \ell-2} \alpha_{2 \ell-1}} \\
& \times \frac{\left(x_{12} x_{23} i \sigma_{2}\right)^{\alpha(\beta}\left(x_{31} x_{12} x_{23} i \sigma_{2}\right)^{\alpha_{1} \alpha_{2}} \cdots\left(x_{31} x_{12} x_{23} i \sigma_{2}\right)^{\left.\alpha_{2 \ell-2} \alpha_{2 \ell-1}\right)}}{\left|x_{12}\right|^{-\Delta+\ell+\frac{1}{2}}\left|x_{23}\right|^{\Delta-\Delta_{12}+\ell+\frac{1}{2}}\left|x_{31}\right|^{\Delta+\Delta_{12}+\ell-\frac{1}{2}}\left|x_{34}\right|^{-\Delta+\ell-\frac{1}{2}}}
\end{aligned}
$$

It is sufficient to write these expressions for a convenient choice of the 4 coordinates $x_{i}$ :

$$
\begin{aligned}
& x_{1}=(0,-1,0), \\
& x_{2}=(0,1,0), \\
& x_{3}=(0, r \cos \theta, r \sin \theta), \\
& x_{4}=(0,-r \cos \theta,-r \sin \theta) .
\end{aligned}
$$

With this choice, the OPE limit $x_{3} \rightarrow x_{4}$ is realized as $r \rightarrow 0$. In this limit, the structures that appear in (B.6)-(B.9) can be approximated as

$$
\begin{aligned}
i \sigma_{2} x_{34} & \approx-2 r\left(-\sigma_{3} \cos \theta+\sigma_{1} \sin \theta\right), & x_{23} i \sigma_{2} & \approx-\sigma_{3}, \\
x_{12} x_{23} i \sigma_{2} & \approx 2 i \sigma_{2}, & x_{31} x_{12} x_{23} i \sigma_{2} & \approx 2 \sigma_{3},
\end{aligned}
$$

while $\left|x_{12}\right| \approx 2,\left|x_{34}\right| \approx 2 r, x_{14} \approx x_{23} \approx \sqrt{1+r^{2}-2 r \cos \theta}$, and $x_{24} \approx x_{13} \approx$ $\sqrt{1+r^{2}+2 r \cos \theta}$. Thus (B.6)-(B.9) become

$$
\begin{aligned}
& \left(g_{\mathcal{O}}^{++}\right)^{\alpha \beta}\left(x_{i}\right) \approx(-1)^{\ell+1} \frac{(4 r)^{\Delta}}{c_{\mathcal{O}}}\left(M_{\theta}\right)_{\alpha_{1} \alpha_{2}} \cdots\left(M_{\theta}\right)_{\alpha_{2 l-2} \alpha_{2 l-1}}\left(-\sigma_{3}\right)^{\alpha(\beta} M_{0}^{\alpha_{1} \alpha_{2}} \cdots M_{0}^{\left.\alpha_{2 l-2} \alpha_{2 l-1}\right)}, \\
& \left(g^{--}\right)_{\mathcal{O}}^{\alpha \beta}\left(x_{i}\right) \approx(-1)^{\ell} \frac{(4 r)^{\Delta}}{c_{\mathcal{O}}}\left(M_{\theta}\right)_{\alpha_{1} \alpha_{2}} \cdots\left(M_{\theta}\right)_{\alpha_{2 l-2} \alpha_{2 l-1}}\left(\tilde{M}_{\theta}\right)_{\gamma}^{\beta}\left(-i \sigma_{2}\right)^{\alpha(\gamma} M_{0}^{\alpha_{1} \alpha_{2}} \cdots M_{0}^{\left.\alpha_{2 l-2} \alpha_{2 l-1}\right)},
\end{aligned}
$$




$$
\begin{aligned}
& \left(g^{+-}\right)_{\mathcal{O}}^{\alpha \beta}\left(x_{i}\right) \approx(-1)^{\ell+1} \frac{(4 r)^{\Delta}}{c_{\mathcal{O}}}\left(M_{\theta}\right)_{\alpha_{1} \alpha_{2}} \cdots\left(M_{\theta}\right)_{\alpha_{2 l-2} \alpha_{2 l-1}}\left(\tilde{M}_{\theta}\right)_{\gamma}^{\beta}\left(-\sigma_{3}\right)^{\alpha(\gamma} M_{0}^{\alpha_{1} \alpha_{2}} \cdots M_{0}^{\left.\alpha_{2 l-2} \alpha_{2 l-1}\right)}, \\
& \left.\left(g^{-+}\right)_{\mathcal{O}}^{\alpha \beta}\left(x_{i}\right) \approx(-1)^{\ell} \frac{(4 r)^{\Delta}}{c_{\mathcal{O}}}\left(M_{\theta}\right)_{\alpha_{1} \alpha_{2}} \cdots\left(M_{\theta}\right)_{\alpha_{2 l-2} \alpha_{2 l-1}}\left(-i \sigma_{2}\right)^{\alpha(\beta} M_{0}^{\alpha_{1} \alpha_{2}} \cdots M_{0}^{\left.\alpha_{2 l-2} \alpha_{2 l-1}\right)}, \quad \text { B. } 12\right)
\end{aligned}
$$

where $M_{\theta}=\sigma_{3} \cos \theta-\sigma_{1} \sin \theta$ and $\tilde{M}_{\theta}=\sigma_{3} \sin \theta+\sigma_{1} \cos \theta$.

This expression should be compared with our 4-point function in a similar limit. Projected down to 3D, the 4-point function (2.12) takes the form:

$$
\left\langle\Psi_{1}^{\alpha}\left(x_{1}\right) \Phi_{2}\left(x_{2}\right) \Phi_{3}\left(x_{3}\right) \Psi_{4}^{\beta}\left(x_{4}\right)\right\rangle=\left(\frac{\left|x_{24}\right|}{\left|x_{14}\right|}\right)^{\Delta_{12}}\left(\frac{\left|x_{13}\right|}{\left|x_{14}\right|}\right)^{\Delta_{43}} \frac{\sum_{I} t_{I}^{\alpha \beta} g^{I}(u, v)}{\left|x_{12}\right|^{\Delta_{1}+\Delta_{2}}\left|x_{34}\right|^{\Delta_{3}+\Delta_{4}}},
$$

with the basis elements being

$$
\begin{aligned}
t_{1}^{\alpha \beta} & =i \frac{\left(x_{14} i \sigma_{2}\right)^{\alpha \beta}}{\left|x_{14}\right|}, \quad t_{2}^{\alpha \beta}=-i \frac{\left(x_{12} x_{23} x_{34} i \sigma_{2}\right)^{\alpha \beta}}{\left|x_{12}\right|\left|x_{23}\right|\left|x_{34}\right|}, \\
t_{3}^{\alpha \beta} & =i \frac{\left(x_{12} x_{24} i \sigma_{2}\right)^{\alpha \beta}}{\left|x_{12}\right|\left|x_{24}\right|}, \quad t_{4}^{\alpha \beta}=i \frac{\left(x_{13} x_{34} i \sigma_{2}\right)^{\alpha \beta}}{\left|x_{13}\right|\left|x_{34}\right|}
\end{aligned}
$$

Considering the point choice (B.10) and taking $r \rightarrow 0$, we have

$$
\begin{aligned}
& t_{1} \approx i \sigma_{3}, \quad t_{2} \approx i\left(-\sigma_{3} \cos \theta+\sigma_{1} \sin \theta\right), \\
& t_{3} \approx \sigma_{2}, \quad t_{4} \approx \sigma_{2} \cos \theta-i \mathbf{1}_{2} \sin \theta .
\end{aligned}
$$

In this limit, the functions appearing in the four-point function (B.13) are simply given $g_{\Delta, \ell}^{I}(r, \eta)=r^{\Delta} h_{\Delta, \ell}^{(\infty) I}(r, \eta)+O\left(r^{\Delta+1}\right)$. Using the first order expansion in $r$ for $h_{\infty, \ell}^{I_{+}}(r, \eta)$ and for $h_{\infty, \ell}^{I_{-}}(r, \eta)(3.1)$ in the limit $r \rightarrow 0$, together with (B.15) and (B.12), we find that in the OPE limit

$$
\begin{aligned}
& \left(\begin{array}{l}
\left(g^{++}\right)_{\mathcal{O}}^{\alpha \beta}\left(x_{i}\right) \\
\left(g^{--}\right)_{\mathcal{O}}^{\alpha \beta}\left(x_{i}\right)
\end{array}\right) \approx \frac{4^{\Delta-1}\left(\ell+\frac{1}{2}\right) !}{\left(\frac{1}{2}\right)_{\ell+\frac{1}{2}}} \frac{1}{c_{\mathcal{O}}} \sum_{I_{+}} t_{I_{+}}^{\alpha \beta} \mathbf{g}_{\Delta, \ell}^{I_{+}}(u, v), \\
& \left(\begin{array}{l}
\left(g^{+-}\right)_{\mathcal{O}}^{\alpha \beta}\left(x_{i}\right) \\
\left(g^{-+}\right)_{\mathcal{O}}^{\alpha \beta}\left(x_{i}\right)
\end{array}\right) \approx \frac{4^{\Delta-1}\left(\ell+\frac{1}{2}\right) !}{\left(\frac{1}{2}\right)_{\ell+\frac{1}{2}}} \frac{1}{c_{\mathcal{O}}} \sum_{I_{-}} t_{I_{-}}^{\alpha \beta} \mathbf{g}_{\Delta, \ell}^{I_{-}}(u, v),
\end{aligned}
$$

where $\mathbf{g}_{\Delta, \ell}^{I} \approx r^{\Delta} \mathbf{h}_{\infty, \ell}^{I}$ with $\mathbf{h}_{\infty, \ell}^{I}$ given by (3.1). In order for the OPE coefficients appearing in the three (2.7) and four-point (2.17) functions to be identical, we must choose

$$
c_{\mathcal{O}}=\frac{4^{\Delta-1}\left(\ell+\frac{1}{2}\right) !}{\left(\frac{1}{2}\right)_{\ell+\frac{1}{2}}}
$$

Open Access. This article is distributed under the terms of the Creative Commons Attribution License (CC-BY 4.0), which permits any use, distribution and reproduction in any medium, provided the original author(s) and source are credited. 


\section{References}

[1] S. Ferrara, A.F. Grillo and R. Gatto, Tensor representations of conformal algebra and conformally covariant operator product expansion, Annals Phys. 76 (1973) 161 [INSPIRE].

[2] A.M. Polyakov, Nonhamiltonian approach to conformal quantum field theory, Zh. Eksp. Teor. Fiz. 66 (1974) 23 [INSPIRE].

[3] G. Mack, Duality in quantum field theory, Nucl. Phys. B 118 (1977) 445 [INSPIRE].

[4] R. Rattazzi, V.S. Rychkov, E. Tonni and A. Vichi, Bounding scalar operator dimensions in $4 D$ CFT, JHEP 12 (2008) 031 [arXiv:0807.0004] [INSPIRE].

[5] V.S. Rychkov and A. Vichi, Universal constraints on conformal operator dimensions, Phys. Rev. D 80 (2009) 045006 [arXiv:0905.2211] [InSPIRE].

[6] F. Caracciolo and V.S. Rychkov, Rigorous limits on the interaction strength in quantum field theory, Phys. Rev. D 81 (2010) 085037 [arXiv:0912.2726] [INSPIRE].

[7] D. Poland and D. Simmons-Duffin, Bounds on $4 D$ conformal and superconformal field theories, JHEP 05 (2011) 017 [arXiv: 1009.2087] [INSPIRE].

[8] R. Rattazzi, S. Rychkov and A. Vichi, Central charge bounds in $4 D$ conformal field theory, Phys. Rev. D 83 (2011) 046011 [arXiv: 1009.2725] [InSPIRE].

[9] R. Rattazzi, S. Rychkov and A. Vichi, Bounds in $4 D$ conformal field theories with global symmetry, J. Phys. A 44 (2011) 035402 [arXiv: 1009.5985] [InSPIRE].

[10] A. Vichi, Improved bounds for CFT's with global symmetries, JHEP 01 (2012) 162 [arXiv:1106.4037] [INSPIRE].

[11] D. Poland, D. Simmons-Duffin and A. Vichi, Carving out the space of $4 D$ CFTs, JHEP 05 (2012) 110 [arXiv:1109.5176] [INSPIRE].

[12] S. Rychkov, Conformal bootstrap in three dimensions?, arXiv:1111.2115 [INSPIRE].

[13] S. El-Showk, M.F. Paulos, D. Poland, S. Rychkov, D. Simmons-Duffin and A. Vichi, Solving the 3D Ising model with the conformal bootstrap, Phys. Rev. D 86 (2012) 025022 [arXiv: 1203.6064] [INSPIRE].

[14] P. Liendo, L. Rastelli and B.C. van Rees, The bootstrap program for boundary CFT , $_{\text {, }}$ JHEP 07 (2013) 113 [arXiv: 1210.4258] [INSPIRE].

[15] C. Beem, L. Rastelli and B.C. van Rees, The $N=4$ superconformal bootstrap, Phys. Rev. Lett. 111 (2013) 071601 [arXiv:1304.1803] [INSPIRE].

[16] F. Kos, D. Poland and D. Simmons-Duffin, Bootstrapping the $O(N)$ vector models, JHEP 06 (2014) 091 [arXiv:1307.6856] [INSPIRE].

[17] S. El-Showk, M. Paulos, D. Poland, S. Rychkov, D. Simmons-Duffin and A. Vichi, Conformal field theories in fractional dimensions, Phys. Rev. Lett. 112 (2014) 141601 [arXiv: 1309.5089] [INSPIRE].

[18] L.F. Alday and A. Bissi, The superconformal bootstrap for structure constants, JHEP 09 (2014) 144 [arXiv: 1310.3757] [INSPIRE].

[19] D. Gaiotto, D. Mazac and M.F. Paulos, Bootstrapping the $3 d$ Ising twist defect, JHEP 03 (2014) 100 [arXiv:1310.5078] [INSPIRE].

[20] D. Bashkirov, Bootstrapping the $N=1$ SCFT in three dimensions, arXiv:1310.8255 [INSPIRE]. 
[21] M. Berkooz, R. Yacoby and A. Zait, Bounds on $N=1$ superconformal theories with global symmetries, JHEP 08 (2014) 008 [Erratum ibid. 01 (2015) 132] [arXiv:1402.6068] [INSPIRE].

[22] S. El-Showk, M.F. Paulos, D. Poland, S. Rychkov, D. Simmons-Duffin and A. Vichi, Solving the $3 d$ Ising model with the conformal bootstrap II. c-minimization and precise critical exponents, J. Stat. Phys. 157 (2014) 869 [arXiv:1403.4545] [INSPIRE].

[23] Y. Nakayama and T. Ohtsuki, Approaching the conformal window of $O(N) \times O(M)$ symmetric Landau-Ginzburg models using the conformal bootstrap, Phys. Rev. D 89 (2014) 126009 [arXiv: 1404.0489] [InSPIRE].

[24] Y. Nakayama and T. Ohtsuki, Five dimensional $O(N)$-symmetric CFTs from conformal bootstrap, Phys. Lett. B 734 (2014) 193 [arXiv:1404.5201] [INSPIRE].

[25] L.F. Alday and A. Bissi, Generalized bootstrap equations for $N=4$ SCFT, JHEP 02 (2015) 101 [arXiv:1404.5864] [INSPIRE].

[26] S.M. Chester, J. Lee, S.S. Pufu and R. Yacoby, The $N=8$ superconformal bootstrap in three dimensions, JHEP 09 (2014) 143 [arXiv: 1406.4814] [INSPIRE].

[27] F. Kos, D. Poland and D. Simmons-Duffin, Bootstrapping mixed correlators in the $3 D$ Ising model, JHEP 11 (2014) 109 [arXiv:1406.4858] [INSPIRE].

[28] F. Caracciolo, A.C. Echeverri, B. von Harling and M. Serone, Bounds on OPE coefficients in $4 D$ conformal field theories, JHEP 10 (2014) 020 [arXiv: 1406.7845] [INSPIRE].

[29] Y. Nakayama and T. Ohtsuki, Bootstrapping phase transitions in QCD and frustrated spin systems, Phys. Rev. D 91 (2015) 021901 [arXiv: 1407.6195] [INSPIRE].

[30] J. Golden and M.F. Paulos, No unitary bootstrap for the fractal Ising model, JHEP 03 (2015) 167 [arXiv:1411.7932] [INSPIRE].

[31] S.M. Chester, J. Lee, S.S. Pufu and R. Yacoby, Exact correlators of BPS operators from the 3d superconformal bootstrap, JHEP 03 (2015) 130 [arXiv:1412.0334] [INSPIRE].

[32] M.F. Paulos, JuliBootS: a hands-on guide to the conformal bootstrap, arXiv:1412.4127 [INSPIRE].

[33] C. Beem, M. Lemos, P. Liendo, L. Rastelli and B.C. van Rees, The $N=2$ superconformal bootstrap, arXiv: 1412.7541 [INSPIRE].

[34] D. Simmons-Duffin, A semidefinite program solver for the conformal bootstrap, JHEP 06 (2015) 174 [arXiv: 1502.02033] [INSPIRE].

[35] N. Bobev, S. El-Showk, D. Mazac and M.F. Paulos, Bootstrapping the three-dimensional supersymmetric Ising model, Phys. Rev. Lett. 115 (2015) 051601 [arXiv:1502.04124] [INSPIRE].

[36] N. Bobev, S. El-Showk, D. Mazac and M.F. Paulos, Bootstrapping SCFTs with four supercharges, JHEP 08 (2015) 142 [arXiv:1503.02081] [INSPIRE].

[37] F. Kos, D. Poland, D. Simmons-Duffin and A. Vichi, Bootstrapping the $O(N)$ archipelago, JHEP 11 (2015) 106 [arXiv:1504.07997] [INSPIRE].

[38] S.M. Chester, S. Giombi, L.V. Iliesiu, I.R. Klebanov, S.S. Pufu and R. Yacoby, Accidental symmetries and the conformal bootstrap, JHEP 01 (2016) 110 [arXiv:1507.04424] [INSPIRE]. 
[39] C. Beem, M. Lemos, L. Rastelli and B.C. van Rees, The $(2,0)$ superconformal bootstrap, Phys. Rev. D 93 (2016) 025016 [arXiv: 1507.05637] [InSPIRE].

[40] L. Iliesiu, F. Kos, D. Poland, S.S. Pufu, D. Simmons-Duffin and R. Yacoby, Bootstrapping $3 D$ fermions, JHEP 03 (2016) 120 [arXiv:1508.00012] [INSPIRE].

[41] D. Poland and A. Stergiou, Exploring the minimal 4D N=1 SCFT, JHEP 12 (2015) 121 [arXiv: 1509.06368] [INSPIRE].

[42] M. Lemos and P. Liendo, Bootstrapping $N=2$ chiral correlators, JHEP 01 (2016) 025 [arXiv: 1510.03866$]$ [INSPIRE].

[43] F.A. Dolan and H. Osborn, Conformal four point functions and the operator product expansion, Nucl. Phys. B 599 (2001) 459 [hep-th/0011040] [INSPIRE].

[44] F.A. Dolan and H. Osborn, Conformal partial waves and the operator product expansion, Nucl. Phys. B 678 (2004) 491 [hep-th/0309180] [INSPIRE].

[45] F.A. Dolan and H. Osborn, Conformal partial waves: further mathematical results, arXiv:1108.6194 [INSPIRE].

[46] M.S. Costa, J. Penedones, D. Poland and S. Rychkov, Spinning conformal blocks, JHEP 11 (2011) 154 [arXiv:1109.6321] [INSPIRE].

[47] A.C. Echeverri, E. Elkhidir, D. Karateev and M. Serone, Deconstructing conformal blocks in $4 D$ CFT, JHEP 08 (2015) 101 [arXiv: 1505. 03750] [INSPIRE].

[48] D. Simmons-Duffin, Projectors, shadows and conformal blocks, JHEP 04 (2014) 146 [arXiv: 1204.3894] [INSPIRE].

[49] M.S. Costa and T. Hansen, Conformal correlators of mixed-symmetry tensors, JHEP 02 (2015) 151 [arXiv: 1411.7351] [INSPIRE].

[50] F. Rejon-Barrera and D. Robbins, Scalar-vector bootstrap, JHEP 01 (2016) 139 [arXiv: 1508.02676] [INSPIRE].

[51] M. Hogervorst and S. Rychkov, Radial coordinates for conformal blocks, Phys. Rev. D 87 (2013) 106004 [arXiv: 1303.1111] [INSPIRE].

[52] J. Penedones, E. Trevisani and M. Yamazaki, Recursion relations for conformal blocks, arXiv:1509.00428 [INSPIRE]. 